\title{
Targeted temperature management for ischemic stroke
}

\section{REVIEW ARTICLE}

Ji Man Hong, MD, PhD

Department of Neurology, Ajou University School of Medicine, Suwon, Republic of Korea
Journal of

Neurocritical

\section{Care}

\author{
Received: October 5, 2019 \\ Revised: November 29, 2019 \\ Accepted: November 29, 2019 \\ Corresponding Author: \\ Ji Man Hong, MD, PhD \\ Department of Neurology, Ajou \\ University School of Medicine, 164 \\ World cup-ro, Yeongtong-gu, Suwon \\ 16499, Republic of Korea \\ Tel: +82-31-219-5175 \\ Fax: +82-31-219-5178 \\ E-mail: dacda@hanmail.net
}

Therapeutic hypothermia (TH) or targeted temperature management is an intentional cooling or temperature control technique using a thermostatic equipment (i.e., surface, endovascular, and focal devices) for specific therapeutic purposes. Given the modifiable determinants in the therapeutic process of acute ischemic stroke, hemorrhagic stroke, and subarachnoid hemorrhage, several studies strongly reported that fever is closely associated with more harmful neurological outcomes. Fever or hyperthermia can deteriorate the cellular ischemic cascade, causing more cerebral damage owing to increased metabolic demands, enhanced release of excitatory neurotransmitters, and increased toxic free radical production. Despite the disappointing results of various clinical trials on TH in the stroke field, many studies have repeatedly clarified its fundamental mechanisms, which proved its strong neuroprotection in experimental stroke models. In particular, the potential effectiveness of TH can be revisited in the era of endovascular thrombectomy for patients undergoing emergent large-vessel occlusion because neuroprotection is maximized in ischemia-reperfusion injury models. This review aims to incorporate current literature into risks and benefits in patients with ischemic stroke.

Keywords: Hypothermia; Hypothermia, induced; Reperfusion injury; Reperfusion

\section{INTRODUCTION}

Stroke is a heterogeneous syndrome caused by the disruption of cerebral blood flow with subsequent tissue damage [1]. Epidemiologically, it is a serious neurological disease considered as the major cause of death and disability worldwide $[2,3]$. It can be classified into ischemic or hemorrhagic, with $85 \%$ of strokes being ischemic, an episode of neurological dysfunction caused by vascular stenosis or occlusion within a specific vascular territory [4].

Despite making up to only $2 \%$ of the total body weight, the brain consumes $20 \%$ of the body's total energy and relies on a constant supply of glucose and oxygen to maintain its function and structural integrity [1]. Therefore, focal cerebral ischemia with severe hypoperfusion falling below the infarct threshold triggers a cascade of ischemic injury [5]. Therapeutic hypothermia $(\mathrm{TH})$, which prevents irreversible neuronal necrosis and cerebral infarction, has strongly been investigated in animal studies and clinical trials with more effectiveness in postcardiac arrest and neonatal encephalopathy, primarily to prevent ischemia-reperfusion injury [6-8].

(C) 2019 The Korean Neurocritical Care Society

This is an Open Access article distributed under the terms of the Creative Commons Attribution Non-Commercial License (http://creativecommons.org/licenses/by-nc/4.0/) which permits unrestricted noncommercial use, distribution, and reproduction in any medium, provided the original work is properly cited. 


\section{MECHANISMS OF ACTION OF THERAPEUTIC HYPOTHERMIA IN ACUTE ISCHEMIC INJURY}

The brain injury mechanism after an ischemic stroke refers to the interaction of complex pathophysiological processes such as excitotoxicity, inflammatory pathways, oxidative damage, blood-brain barrier disruption, angiogenesis, and its restoration [4]. Current neuroprotection treatments for ischemic stroke injuries are not proven beneficial in the case of cerebral ischemia due to the complexity and disappointing results of various drug experiments in human clinical trials [9]. In contrast, TH is believed to inhibit or at least reduce the progression of this cascade at multiple levels (Fig. 1). Ischemic injury cascade starts with cerebral hypoxia, resulting in a loss of adenosine triphosphate (ATP) production and dysfunction of ATP-dependent $\mathrm{Na}^{+}-\mathrm{K}^{+}$pumps in the cell membrane. The key cascades of this pathological process also commonly occur in various ischemic strokes. While the precise mechanism has not been fully understood, numerous hypotheses have suggested the neuroprotective effects of $\mathrm{TH}$ such as prevention of blood-brain barrier disruption; reduction of cerebral glucose metabolism and oxygen consumption; reduction of excitotoxic neurotransmitter accumulation, intracellular acidosis, intracellular calcium influx, and oxygen-free radical production; alteration of cold shock protein expression; reduction of brain edema; reduction of thrombosis risk; and reduction of the risk of epileptic activities [10]. In addition, TH can function as an antiedema therapy to prevent the increase in intracerebral pressure or impending cerebral herniation during an acute period of ischemic stroke [11]. In summary, potential mechanisms of TH can be described as "neuroglial protectors" for acute ischemic stroke.

\section{COMPLICATIONS OF THERAPEUTIC HYPOTHERMIA}

As mild hypothermia $\left(34^{\circ} \mathrm{C}\right.$ to $\left.35.9^{\circ} \mathrm{C}\right)$ is relatively well tolerated, deep hypothermia $\left(<32^{\circ} \mathrm{C}\right)$ appears to be related to more delete-

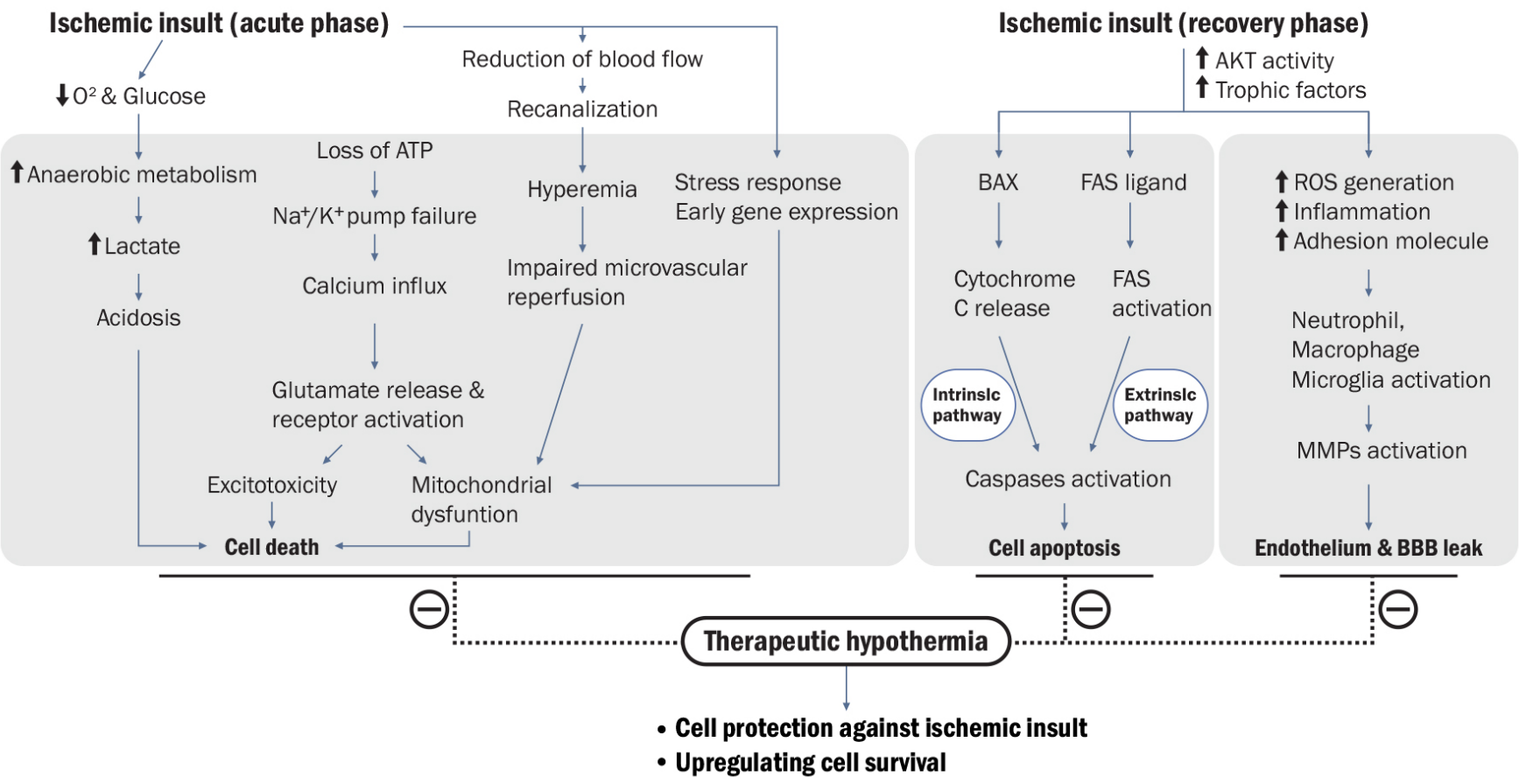

Fig. 1. Possible tissue damage mechanisms that therapeutic hypothermia $(\mathrm{TH})$ can alleviate in ischemic stroke from acute stage to recovery phase [10]. (A) Acute events, such as loss of oxygen and glucose after stroke, can lead to energy loss (adenosine triphosphate [ATP] loss) and ion pump failure. The resulting loss of concentration gradients allows ions $\left(\mathrm{Na}^{+} / \mathrm{K}^{+}\right)$to flow down their concentration gradients, leading to cellular swelling (cytotoxic edema) and release of excitatory amino acids. Reperfusion injury after recanalization may also occur, and tissue hypoxia can increase anaerobic metabolism. All of these events will lead to acute cell death. TH can alleviate almost all ischemic cascades that lead to cell death. (B) There are possible mechanisms that TH can alleviate the recovery phase of ischemic stroke. Ischemia activates both intrinsic and extrinsic pathways that will lead to cell apoptosis. It also triggers reactive oxygen species (ROS) production and inflammatory reaction. These promote endothelial cell damage and blood-brain barrier (BBB) disruption. As a result, TH has been associated with suppression of both apoptotic and inflammatory pathways, while upregulating cell survival pathways. BAX, Bcl2 associated X protein; MMP, matrix metalloprotelnase. 
rious side effects directly caused by the intervention itself [12]. Physiological changes should be carefully considered and closely monitored on a daily basis in intensive care units (ICUs), and patients considering TH should be admitted to the ICU $[13,14]$.

Side effects of TH can be categorized as cardiac, hematologic, immunologic, and metabolic complications [14,15]. Fig. 2 shows examples of complications during induction, maintenance, and rewarming of TH. Shivering in response to hypothermia can undermine the intentional goal of therapeutic cooling by generating heat that leads to increased core temperature and oxygen consumption. It is most prominent in the $\mathrm{TH}$ induction process; therefore, the use of sedatives and paralytics during this period should be carefully considered [16].

\section{PREVIOUS STUDIES ON ACUTE ISCHEMIC STROKE}

Several small phase II pilot trials have investigated the safety and feasibility of TH for acute ischemic stroke. These included the Cooling for Acute Ischemic Brain Damage (COOL-AID) trial [17]. This endovascular cooling trial demonstrated positive results of slower infarct growth in the TH group compared to that in the control group. As a result, the Intravascular Cooling in the treatment of stroke longer tissue plasminogen activator (tPA) window (ICTuS-L) was a phase I trial originally designed to establish the safety of endovascular cooling combined with tPA. Unfortunately, this trial was an eventual failure owing to a significantly increased proportion of pneumonia cases in the TH group [18].

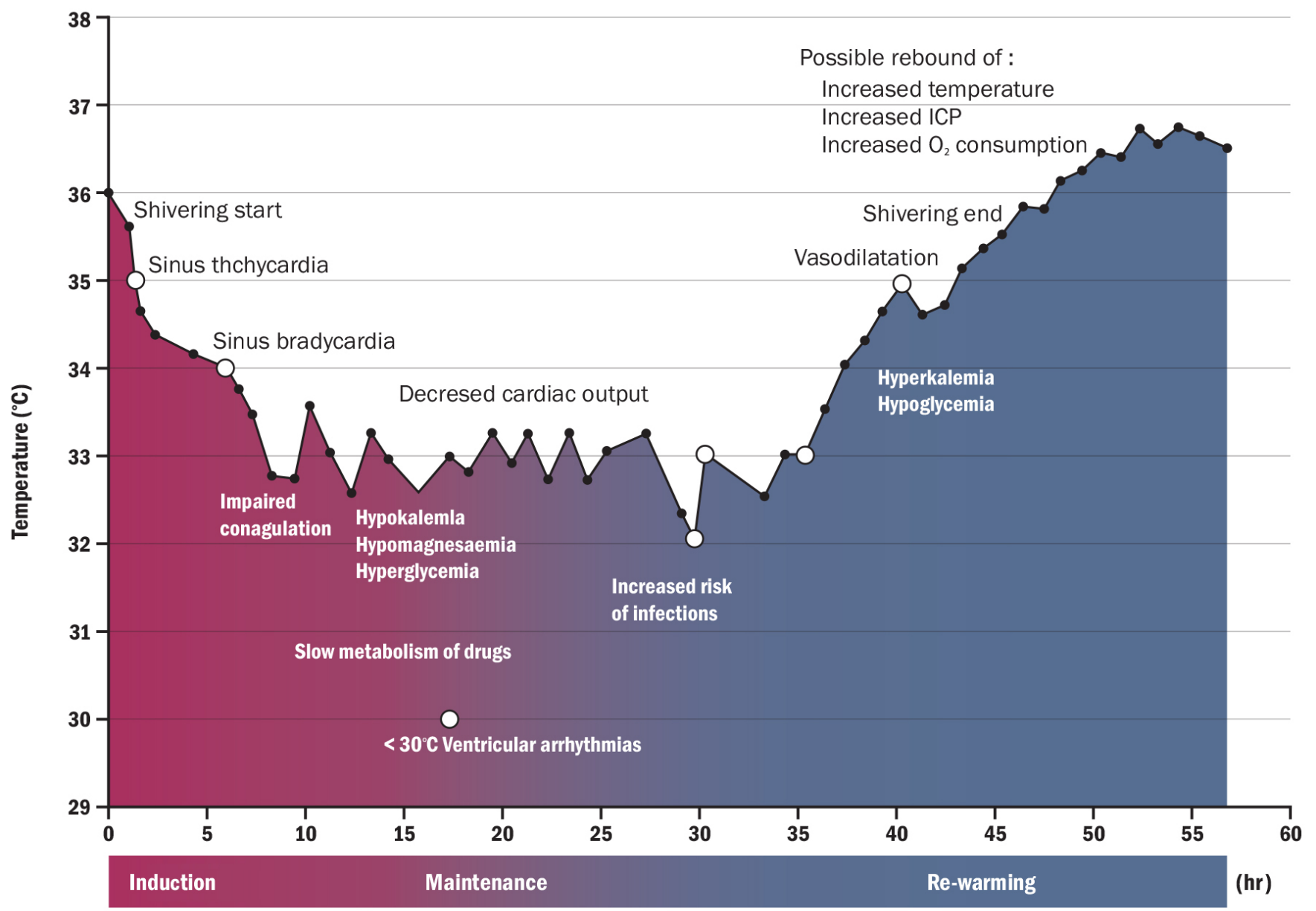

Fig. 2. Physiological changes (open circle) according to temperature variation and possible complications (closed circle) during the three phases of therapeutic hypothermia: induction, maintenance, and rewarming [14]. Possible cooling complications may present as reverse patterns of rewarming complications (hyperkalemia, hypoglycemia, and rebound of increased intracranial pressure, among others). ICP, intracerebral pressure. 
For safety procedures and faster cooling than that in the previous ICTuS-L trial, the protocol of ICTuS 2 trial was modified in a prospective, multisite phase $2 / 3$ pivotal, tissue plasminogen activator (tPA) trial combined with intravascular cooling catheter hypothermia in awake patients with moderate-to-severe middle cerebral artery (MCA) infarction with cold saline bolus and permissive hypothermia [19]. Unfortunately, this also did not show the usefulness of TH [20]. Recently, a phase III trial, an European randomized open-label clinical investigation with blinded outcome assessment (EuroHYP-1), provided no evidence that active cooling to a target of $34.0^{\circ} \mathrm{C}$ to $35.0^{\circ} \mathrm{C}$ for 12 to 24 hours initiated within 6 hours after the onset of ischemic stroke has an impact on the functional outcomes at 3 months [21]. This trial was discontinued after including 98 of the originally intended 1,500 patients because of slow recruitment and cessation of funding. This trial also was substantially underpowered to detect any clinically relevant benefit or harm [21]. Moreover, ICTuS 3 study poorly detected the clinical benefits owing to issues in patient enrollment during the study. These studies also raised questions about the feasibility of inducing hypothermia in relatively awake, spontaneously breathing stroke patients who tend to shiver vigorously and are prone to experience discomfort with hypothermia (unlike intubated and cardiac arrest patients).

Decompressive hemicraniectomy has been considered a powerful beneficial option to reduce the mortality of severely ill patients with a space-occupying MCA infarction; however, a considerable number of patients are still suffering from serious disability or even face death. Therefore, TH can be clinically beneficial in those with severe stroke treated with hemicraniectomy because of its strong antiedema therapy during the acute phase of ischemic stroke. Nonetheless, several clinical data demonstrated that hypothermia treatment had no additional benefits on the functional outcome as compared with hemicraniectomy alone [11,22,23]. Therefore, we should include specific candidates who would be more feasible targets for detecting the clinical efficacy and safety of TH in future trials.

\section{COMPARATIVE LESSONS ON THERAPEUTIC HYPOTHERMIA: SUCCESSFUL GLOBAL ISCHEMIA VS. FAILED FOCAL ISCHEMIA}

Since 2002, when the trial "Hypothermia after Cardiac Arrest (HACA)" study group demonstrated the clinical benefits of TH in improving neurological and mortality outcomes in postcardiac arrest patients with shockable rhythms, many studies have shown the positive effects of hypothermia on the neuronal protection in global brain ischemia $[6,7]$.
However, the evidence of $\mathrm{TH}$ for focal cerebral ischemia in stroke remains inconclusive. Preclinical studies support that TH is much more beneficial and consistent in temporary MCA occlusion than in permanent MCA models [24]. In addition, several studies reported that despite the association of $\mathrm{TH}$ with increased risk of pneumonia, longer duration of ICU stay, and prolonged mechanical ventilation dependency, these factors did not affect the neurological outcome and ICU survival [25]. Compared to the conventional TH guideline on postcardiac arrest syndrome, some data showed a beneficial tendency in case of longer duration of cooling and rewarming in cardiac arrest [26], ischemic stroke [27], and traumatic brain injury [28]. Generally, clinical deterioration after an ischemic stroke attributable to $\mathrm{HT}$ and cerebral edema usually occurs between 2 and 5 days after stroke [29]. The time course of edema after stroke and extrapolation from traumatic brain injury hypothermia studies suggesting a prolonged course of $\mathrm{TH}$ with slow and controlled rewarming may be important for the success of TH protocols in patients with stroke. Therefore, animal and clinical studies suggest that $\mathrm{TH}$ with a long-durational and specific protocol might be more effective in patients with severe stroke who underwent successful recanalization by preventing ischemia-reperfusion injury in endotracheal intubation obligation such as cardiac arrest or perinatal postanoxic status.

\section{LESSONS FROM PREVIOUS IN VIVO EXPERIMENTS}

Although some trials showed positive efficacy of longer TH duration from different clinical situations, its optimal duration remains unclear. Considering that neurovascular damage after an acute ischemic attack occurs over hours to days, the longer the duration of hypothermia is, the more significantly improved the outcomes can be. In support of this hypothesis, some experiments have already demonstrated better outcomes with longer duration of cooling [10]. Interestingly, van der Worp et al. [30] concretely reported an inverse relationship between the duration of $\mathrm{TH}$ and infarct volume in a systemic review and meta-analysis of animal models. As compared to shorter ( 3 hours) periods of hypothermia in Sprague-Dawley rats with transient middle cerebral artery occlusion (tMCAO), infarct size reduction was greater and better outcomes were observed in patients treated in longer hours (21 hours) [31]. This can lead to an expansion of time window and number of patients for the treatment of endovascular recanalization in patients who had emergent large vessel occlusion (ELVO) stroke. Although no guidelines have recommended the exact therapeutic timeframe for TH, time-sensitive characteristics of hypothermia induction cannot be easily disputed due to solid results of 
previous experiments [32]. In summary, available experimental data showed that TH could be (1) more successful when applied quickly; (2) when applied with sufficient duration; and (3) when applied to the ischemia-reperfusion model.

\section{TARGETED TEMPERATURE MANAGEMENT DURING OR AFTER AN ENDOVASCULAR RECANALIZATION}

Endovascular treatment (EVT) such as mechanical clot retrieval has become a proven therapeutic strategy for acute ischemic stroke with an ELVO recently [33]. Many of these patients are at high risk of brain injury owing to their large stroke volume and of reperfusion injury even if recanalization was successful. Among the neuroprotective strategies, $\mathrm{TH}$ has been attempted to evaluate the outcome benefits in experiments and clinical trials $[4,9]$. In a recent postreperfusion TH study, several patients (approximately 45\%) had favorable outcomes based on the modified Rankin Scale $0,1,2$ at 3 months despite low baseline alberta stroke pro- gram early CT score (ASPECTS) and large lesion volume (> 80 $\mathrm{mL}$ ) [34]. Recent EVT trials showed that EVT was useful for patients with a large core volume, and reperfusion rate was a predictor of good outcomes [35-37]. However, several studies in patients with malignant MCA trait demonstrated that low ASPECTS on computed tomography (initially large parenchymal lesion) was a predictor of poor outcomes despite successful recanalization. Such contradictory results demonstrate that reperfusion itself does not always guarantee a good functional outcome, especially in patients with "malignant MCA infarction trait" $[33,38]$. Neuroprotection after recanalization is evident in the EVT era to improve the clinical outcomes of these contradictory groups. Failure of previous neuroprotective drugs can be overcome by achieving endovascular recanalization, a similar mode of ischemic-reperfusion model in preclinical experiments. In this context, immediate postreperfusion cooling can be a promising option to minimize reperfusion-related complications. The rate of infarct growth has also been known to vary depending on an individual's diversity, such as the collateral blood flow in patients with acute ischemic
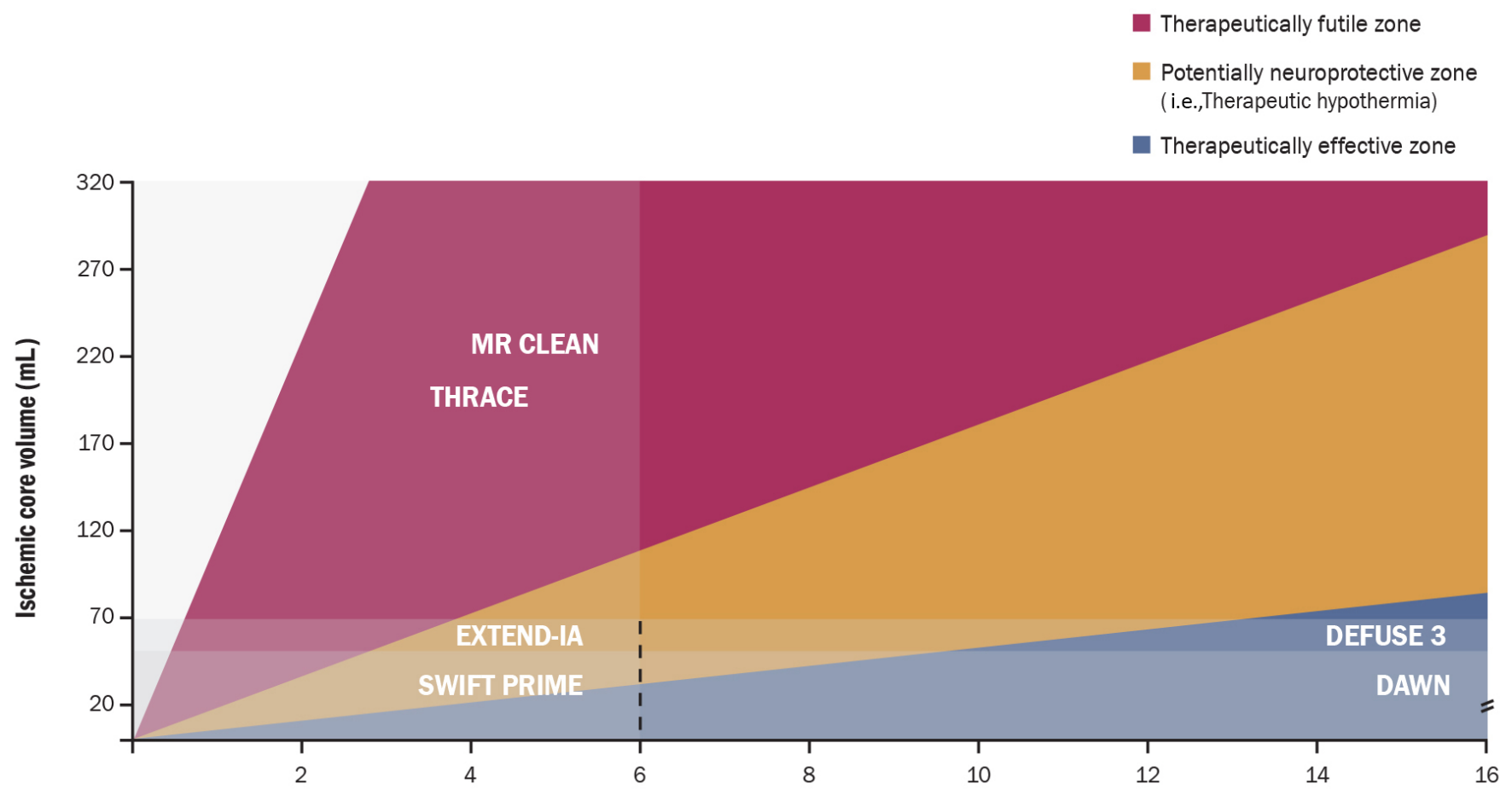

Time between symptom onset and thrombectomy (hr)

Fig. 3. A simplified diagram of the symptom onset, infarct core volume, and potential neuroprotectant (i.e., therapeutic hypothermia) according to the inclusion criteria described in previous successful endovascular treatment trials.35 The red zone represents the fast progressor of the infarct core, the yellow zone the intermediate progressor, and the green zone the slow progressor. Reduction of reperfusion injury can be more effective in patients who are not classified as either fast or slow progressors, such as those in the yellow zone (potentially neuroprotective zone). However, massive reperfusion injury can easily occur in the red zone area (therapeutically futile zone). 
stroke. Recent success of the late window EVT trials may be because of the selection of "slow progressors" in the infarct growth process [35] In the future, neuroprotective modalities, including $\mathrm{TH}$ and other agents for reperfusion injury, may be promising for patients not classified as either fast and slow progressors (Fig. 3). A recent study has shown that neurocritical care treatments including $\mathrm{TH}$ are feasible even in patients with a malignant infarct core [39]. Therefore, the strategy reducing reperfusion injury using $\mathrm{TH}$ will be another option for vulnerable patients suffering from acute ischemic stroke in the EVT era. Although TH protocols remain debatable [40], it can be com bined with existing therapies to improve outcomes of patients with acute ischemic stroke through the technological development of EVT.

\section{CONCLUSION}

TH can play a pivotal role in the era of endovascular thrombectomy for patients undergoing emergent large-vessel occlusion because neuro-glial protection can be maximized in ischemia-reperfusion injury model.

\section{ARTICLE INFORMATION}

\section{Conflict of interest}

No potential conflict of interest relevant to this article.

\section{ORCID}

Ji Man Hong, https://orcid.org/0000-0001-6803-1207

\section{Author contributions}

Conceptualization: JMH. Data curation \& Formal analysis: JMH. Visualization \& Writing-original draft: JMH. Writing-review editing: JMH.

\section{REFERENCES}

1. Markus HS. Cerebral perfusion and stroke.J Neurol Neurosurg Psychiatry 2004;75:353-61.

2. Heron M. Deaths: leading causes for 2004. Natl Vital Stat Rep 2007;56:1-95.

3. Lallukka T, Ervasti J, Lundström E, Mittendorfer-Rutz E, Friberg E, Virtanen M, et al. Trends in diagnosis-specific work disability before and after stroke: a longitudinal population-based study in Sweden.J Am Heart Assoc 2018;7:e006991.

4. Catanese L, Tarsia J, Fisher M. Acute ischemic stroke therapy overview. Circ Res 2017;120:541-58.

5. Hossmann KA. Viability thresholds and the penumbra of focal ischemia. Ann Neurol 1994;36:557-65.

6. Bernard SA, Gray TW, Buist MD, Jones BM, Silvester W, Gutteridge $\mathrm{G}$, et al. Treatment of comatose survivors of out-of-hospital cardiac arrest with induced hypothermia. N Engl J Med 2002;346:557-63.

7. Hypothermia after Cardiac Arrest Study Group. Mild therapeutic hypothermia to improve the neurologic outcome after cardiac arrest. N Engl J Med 2002;346:549-56.

8. Shankaran S. Therapeutic hypothermia for neonatal encephalopathy. Curr Treat Options Neurol 2012;14:608-19.

9. Hong JM, Choi MH, Sohn SI, Hwang YH, Ahn SH, Lee YB, et al. Safety and optimal neuroprotection of neu2000 in acute ischemic stroke with recanalization: study protocol for a randomized, double-blinded, placebo-controlled, phase-II trial. Trials 2018;19:375.

10. Kurisu K, Yenari MA. Therapeutic hypothermia for ischemic stroke: pathophysiology and future promise. Neuropharmacology 2018;134:302-9.

11. Neugebauer H, Schneider H, Bösel J, Hobohm C, Poli S, Kollmar R, et al. Outcomes of hypothermia in addition to decompressive hemicraniectomy in treatment of malignant middle cerebral artery stroke: a randomized clinical trial. JAMA Neurol 2019;76:571-9.

12. Polderman KH, Herold I. Therapeutic hypothermia and controlled normothermia in the intensive care unit: practical considerations, side effects, and cooling methods. Crit Care Med 2009;37:1101-20.

13. Polderman KH. Application of therapeutic hypothermia in the ICU: opportunities and pitfalls of a promising treatment modality. Part 1: indications and evidence. Intensive Care Med 2004;30:556-75.

14. Polderman KH. Mechanisms of action, physiological effects, and complications of hypothermia. Crit Care Med 2009; 37:S186-202.

15. Andresen M, Gazmuri JT, Marín A, Regueira T, Rovegno M. Therapeutic hypothermia for acute brain injuries. Scand J Trauma Resusc Emerg Med 2015;23:42.

16. Badjatia N, Strongilis E, Gordon E, Prescutti M, Fernandez L, Fernandez A, et al. Metabolic impact of shivering during therapeutic temperature modulation: the Bedside Shivering Assessment Scale. Stroke 2008;39:3242-7.

17. De Georgia MA, Krieger DW, Abou-Chebl A, Devlin TG, Jauss M, Davis SM, et al. Cooling for Acute Ischemic Brain Damage (COOL AID): a feasibility trial of endovascular cooling. Neurology 2004;63:312-7.

18. Hemmen TM, Raman R, Guluma KZ, Meyer BC, Gomes JA, Cruz-Flores S, et al. Intravenous thrombolysis plus hypothermia 
for acute treatment of ischemic stroke (ICTuS-L): final results. Stroke 2010;41:2265-70.

19. Lyden PD, Hemmen TM, Grotta J, Rapp K, Raman R. Endovascular therapeutic hypothermia for acute ischemic stroke: ICTuS 2/3 protocol. Int J Stroke 2014;9:117-25.

20. Lyden P, Hemmen T, Grotta J, Rapp K, Ernstrom K, Rzesiewicz $\mathrm{T}$, et al. Results of the ICTuS 2 trial (Intravascular Cooling in the Treatment of Stroke 2). Stroke 2016;47:2888-95.

21. van der Worp HB, Macleod MR, Bath PM, Demotes J, Durand-Zaleski I, Gebhardt B, et al. EuroHYP-1: European multicenter, randomized, phase III clinical trial of therapeutic hypothermia plus best medical treatment vs. best medical treatment alone for acute ischemic stroke. Int J Stroke 2014;9:642-5.

22. Els T, Oehm E, Voigt S, Klisch J, Hetzel A, Kassubek J. Safety and therapeutical benefit of hemicraniectomy combined with mild hypothermia in comparison with hemicraniectomy alone in patients with malignant ischemic stroke. Cerebrovasc Dis 2006;21:79-85.

23. Schneider H, Krüger P, Algra A, Hofmeijer J, van der Worp HB, Jüttler $\mathrm{E}$, et al. No benefits of hypothermia in patients treated with hemicraniectomy for large ischemic stroke. Int J Stroke 2017;12:732-40.

24.Fluri F, Schuhmann MK, Kleinschnitz C. Animal models of ischemic stroke and their application in clinical research. Drug Des Devel Ther 2015;9:3445-54.

25. Perbet S, Mongardon N, Dumas F, Bruel C, Lemiale V, Mourvillier B, et al. Early-onset pneumonia after cardiac arrest: characteristics, risk factors and influence on prognosis. Am J Respir Crit Care Med 2011;184:1048-54.

26. Kirkegaard H, Søreide E, de Haas I, Pettilä V, Taccone FS, Arus $\mathrm{U}$, et al. Targeted temperature management for 48 vs 24 hours and neurologic outcome after out-of-hospital cardiac arrest: a randomized clinical trial. JAMA 2017;318:341-50.

27. Hong JM, Lee JS, Song HJ, Jeong HS, Choi HA, Lee K. Therapeutic hypothermia after recanalization in patients with acute ischemic stroke. Stroke 2014;45:134-40.

28. Jiang JY, Xu W, Li WP, Gao GY, Bao YH, Liang YM, et al. Effect of long-term mild hypothermia or short-term mild hypothermia on outcome of patients with severe traumatic brain injury. J Cereb Blood Flow Metab 2006;26:771-6.

29. Wijdicks EF, Sheth KN, Carter BS, Greer DM, Kasner SE, Kimberly WT, et al. Recommendations for the management of cere- bral and cerebellar infarction with swelling: a statement for healthcare professionals from the American Heart Association/ American Stroke Association. Stroke 2014;45:1222-38.

30. van der Worp HB, Sena ES, Donnan GA, Howells DW, Macleod MR. Hypothermia in animal models of acute ischaemic stroke: a systematic review and meta-analysis. Brain 2007; 130:3063-74.

31. Krieger DW, Yenari MA. Therapeutic hypothermia for acute ischemic stroke: what do laboratory studies teach us? Stroke 2004;35:1482-9.

32. Markarian GZ, Lee JH, Stein DJ, Hong SC. Mild hypothermia: therapeutic window after experimental cerebral ischemia. Neurosurgery 1996;38:542-50.

33. Goyal M, Menon BK, van Zwam WH, Dippel DW, Mitchell PJ, Demchuk AM, et al. Endovascular thrombectomy after large-vessel ischaemic stroke: a meta-analysis of individual patient data from five randomised trials. Lancet 2016;387:172331.

34. Hwang YH, Jeon JS, Kim YW, Kang DH, Kim YS, Liebeskind DS. Impact of immediate post-reperfusion cooling on outcome in patients with acute stroke and substantial ischemic changes. J Neurointerv Surg 2017;9:21-5.

35. Albers GW. Late window paradox. Stroke 2018;49:768-71.

36. Horn CM, Sun CH, Nogueira RG, Patel VN, Krishnan A, Glenn $\mathrm{BA}$, et al. endovascular reperfusion and cooling in cerebral acute ischemia (ReCCLAIM I). J Neurointerv Surg 2014;6:91-5.

37. Rocha M, Jovin TG. Fast versus slow progressors of infarct growth in large vessel occlusion stroke: clinical and research implications. Stroke 2017;48:2621-7.

38. Yoo AJ, Berkhemer OA, Fransen PSS, van den Berg LA, Beumer D, Lingsma HF, et al. Effect of baseline Alberta Stroke Program Early CT Score on safety and efficacy of intra-arterial treatment: a subgroup analysis of a randomised phase 3 trial (MR CLEAN). Lancet Neurol 2016;15:685-94.

39. Lee SJ, Lee KS, Lee JS, Choi MH, Lee SE, Hong JM. Primary neurocritical care involving therapeutic hypothermia for acute ischemic stroke patients with malignant infarct cores. J Neurocrit Care 2019;12:30-6.

40. Kuczynski AM, Demchuk AM, Almekhlafi MA. Therapeutic hypothermia: applications in adults with acute ischemic stroke. Brain Circ 2019;5:43-54. 